\title{
Decreasing environmental tobacco smoke exposure among low income children: preliminary findings
}

\author{
Melbourne F Hovell, Joy M Zakarian, Georg E Matt, C Richard Hofstetter, \\ J Thomas Bernert, James Pirkle
}

Estimates suggest that environmental tobacco smoke (ETS) exposure may affect over $40 \%$ of children in the USA. ${ }^{1}$ ETS exposure is a known health risk, especially for young children. $^{2-5}$ Previous studies have shown that reported ETS exposure can be reduced with counselling. ${ }^{6-8}$ However, no study has demonstrated reduced ETS exposure for low income and minority families, nor have beneficial effects been demonstrated by objective biological measures. Since low income and racial/ethnic minority populations may be at higher risk of exposure as well as at higher risk of inadequate medical/preventive services, testing means of reducing ETS exposure among these populations is critical from a public health and social justice perspective. ${ }^{9}{ }^{10}$ The purpose of this trial was to test a revised in-person and telephone counselling program among low income, multiracial/ethnic families and to assess outcomes based on objective cotinine as well as parent reported exposure measures.

\section{Methods}

Smoking mothers and their babies ( $\mathrm{n}=108$ families) were recruited from San Diego sites providing benefits through the federally funded Special Supplemental Nutrition Program for Women, Infants, and Children (WIC). Screening forms were distributed to all adult clients during a nine month period. Of 1147 mothers who reported that they smoked and had children under the age of 4 years, research assistants were able to screen 832 by telephone. Of these, $162(19.5 \%)$ qualified and were invited to participate in the study. Only $17.9 \%$ refused, and the first 108 who signed informed consents were enrolled. Eligibility criteria included English or Spanish language ability, mothers' reported smoking rate of a minimum of two cigarettes per day with a minimum of one cigarette per day exposure to their baby, access to a telephone, and not breastfeeding (because of confounding of cotinine analyses). ${ }^{11}$

After baseline measures, mothers and babies were assigned at random to counselling or control conditions. Counselling was provided to mothers and sometimes fathers. Counselling involved clinical interviewing, negotiated goals and objectives aimed at reducing babies' exposure, and contingent praise/acknowledgement for successes. Shaping procedures were employed to incrementally move parents to reduce babies' exposure as near to zero as pos- sible. Seven counselling sessions were provided over three months, with a mean duration across sessions of 12.6 to 28 minutes. Counselling session 1 was held at mothers' homes immediately following the baseline interview and random assignment. Sessions 3 and 7 were planned to occur in-person at WIC sites (to coincide with mothers' regularly scheduled appointments when possible), with the remaining sessions delivered via telephone. Only 11 mothers (20.8\%) had two sessions at WIC and 10 mothers (18.9\%) had one. Counsellors met 42 mothers $(79.2 \%)$ at their homes for at least one in-person session, because of transportation, childcare, and other scheduling difficulties. Formal smoking cessation counselling was not provided, although parents who expressed an interest in cessation were encouraged to contact community resources for assistance. Counsellors received 20 hours of training, including role playing, and participated in weekly case review meetings.

Reported ETS exposure, supported by environmental nicotine monitoring ${ }^{12}{ }^{13}$ (including bogus pipeline procedures ${ }^{14}$ ) and babies' urine cotinine assays, were obtained before intervention, at three months post-test, and six (interview only) and 12 months follow up. Babies' urine samples were obtained by placing cotton rolls in babies' diapers, or with a standard urine collection cup for toilet trained babies. Our pilot research has shown that cotton rolls do not alter the concentration of cotinine detected. ${ }^{15}$ Mothers who reported seven day smoking cessation at any measure provided saliva samples for cotinine analysis. Test-retest reliability estimates for reported ETS exposure from mothers and for all sources of ETS exposure were high ( $r=0.91$ and 0.88 ) and significant $(\mathrm{p}<0.05)$. Validity correlations for urine cotinine and environmental nicotine with reported ETS exposure resulted in coefficients that ranged from 0.35 to 0.75 , and reached significance $(p<0.05)$ across groups and measures. Further details regarding the reliability and validity of measures have been previously published. ${ }^{16}$ Separate outcome analyses for reported and biological indicators of ETS exposure will be computed using generalised estimating equations to estimate the degree of differential change.

\section{Preliminary results}

Mothers' mean (SD) age was 28.7 (6.7) years. About half $(47.2 \%)$ were non-Latino white, $27.8 \%$ were Latino, $21.3 \%$ were African Ameri- 
can, and $3.7 \%$ were mixed or other race. Less than high school education was reported by $38.9 \%, 25.0 \%$ reported high school graduation or general equivalency degree, $25.9 \%$ some college, $2.8 \%$ college graduation, and $7.4 \%$ trade school or vocational training. Most mothers $(88.0 \%)$ were not employed, and $46.3 \%$ did not have a husband or other partner living in the home at the baseline interview. Babies' mean (SD) age was 14.2 (6.9) months, and about half $(52.8 \%)$ were female. $\chi^{2}$ and analysis of variance (ANOVA) analyses showed no baseline differences between experimental and control groups in mothers' or babies' age or sex, mothers' ethnicity, education, employment, single parent status, saliva cotinine concentrations, reported smoking rate or lifetime quit attempts, or babies' ETS exposure (based on parent reports or babies' cotinine).

High rates of cohort retention and intervention fidelity were achieved. Forty nine of the 53 $(92.5 \%)$ women assigned to the experimental group completed all seven counselling sessions. One mother skipped her last session because of scheduling difficulties. One moved and could not be located after her third session, and two refused to continue their participation in the study during counselling. Postintervention, seven mothers were lost, one was incarcerated, and one refused to continue. Final 12 month interview measures were obtained from $88.9 \%(n=96)$ of mothers and babies' urine samples were obtained from $87.0 \%(\mathrm{n}=94)$.

Reports of seven day smoking cessation were biochemically verified (saliva cotinine concentration $<30 \mathrm{ng} / \mathrm{ml}$ ) for six mothers in the experimental group and four mothers in the control group at one or more measurement points. This difference did not reach significance $(p>0.05)$.

Results indicated that mothers enjoyed the counselling program and appreciated the assistance. Over half (59.6\%) felt that the advice they received was "very helpful", and all but one of the remaining 19 women felt that it was "somewhat helpful". Almost all (97.9\%) stated that they would recommend this program to a friend, and all mothers felt that WIC should offer this service to families with children exposed to ETS.

Outcome analyses are in progress. If therapeutic effects are observed, these results will add to the literature confirming counselling as a means of reducing children's ETS exposure.

\section{Discussion}

This is one of the few controlled trials of ETS exposure reduction to include a low income and mixed racial/ethnic population, and an objective biological measure of ETS exposure. The low refusal rate and successful program delivery confirmed the feasibility of providing such a service to the WIC population. The findings suggest that families find ETS reduction counselling an aid in their efforts to protect their children from tobacco smoke. Initial reliability and validity estimates of ETS exposure confirm the usefulness of reported measures for both research and future applied services. Initial results suggest that counselling limited to children's ETS exposure does not result in smoking cessation, which indicates that cessation services should be considered in addition to exposure reduction services. Final confirmation of ETS exposure reduction from counselling awaits completion of analyses of both reported and cotinine measures. If results confirm the efficacy of counselling, future studies will be needed to determine possible reductions in morbidity and mortality, and to determine possible costs and cost savings from effective reduction in ETS exposure and related morbidity/medical care services. Finally, incorporation of tobacco control services in the national WIC program deserves further policy consideration.

This research was supported by grant 027946 SFP awarded to Dr Hovell from the Robert Wood Johnson Smoke-Free Families Program.

1 Pirkle JL, Flegal KM, Bernert JT, et al. Exposure of the US population to environmental tobacco smoke. The Third population to environmental tobacco smoke. The Third National Health and Nutrition Exam

2 US Environmental Protection Agency. Respiratory health effects of passive smoking: lung cancer and other disorders. Smoking and Tobacco Control Monograph No 4. Bethesda, Maryland: US Department of Health and Human Services, Public Health Service, National Institutes of Health, 1993. (NIH Publication No 93-3605.)

3 Charlton A. Children and passive smoking: a review. $\mathcal{f}$ Fam Pract 1994;38:267-77.

4 Chilmonczyk BA, Salmun LM, Megathlin KN, et al. Association between exposure to environmental tobacco smoke and exacerbations of asthma in children. $N$ Engl $\mathcal{F}$ Med 1993;328:1665-9.

5 Klonoff-Cohen HS, Edelstein SL, Lefkowitz ES, et al. The effect of passive smoking and tobacco exposure through breast milk on sudden infant death syndrome. $7 A M A$ 1995;273:795-8.

6 Greenberg RA, Strecher VJ, Bauman KE, et al. Evaluation of a home-based intervention program to reduce infant passive smoking and lower respiratory illness. $\mathcal{f}$ Behav Med 1994;17:273-90.

7 Hovell MF, Meltzer SB, Zakarian JM, et al. Reduction of environmental tobacco smoke exposure among asthmatic children: a controlled trial [published erratum appears in Chest 1995;107:480]. Chest 1994;106:440-6.

8 Wahlgren DR, Hovell MF, Meltzer SB, et al. Reduction of environmental tobacco smoke exposure in asthmatic children: a 2-year follow-up. Chest 1997;111:81-8.

9 Kogevinas M, Porta $M$. Socioeconomic differences in cancer survival: a review of the evidence. IARC Sci Publ 1997;138:177-206.

10 Marmot M, Feeney A. General explanations for social inequalities in health. IARC Sci Publ 1997;183:207-28.

11 Schulte-Hobein B, Schwartz-Bickenbach D, Abt S, Plum C, Nau H. Cigarette smoke exposure and development of infants throughout the first year of life: influence of passive smoking and nursing on cotinine levels in breast milk and infant's urine. Acta Paediatr 1992;81:550-7.

12 Hammond SK, Leaderer BP. A diffusion monitor to measure exposure to passive smoking. Environ Sci Tech measure exposure

13 Leaderer BP, Hammond SK. Evaluation of vapor-phase nicotine and respirable suspended particle mass as markers for environmental tobacco smoke. Environ Sci Tech 1991;25:770-7.

14 Murray DM, O'Connell CM, Schmid LA, et al. Validation of smoking by self-report by adolescents: a re-examination of the bogus pipeline procedures. Addict Behav 1987;12:715

15 Matt GE, Wahlgren DR, Hovell MF, et al. Measuring ETS exposure in infants and young children through urine cotinine and memory-based parental reports: empirical findings and discussion. Tobacco Control 1999;8:282-9.

16 Matt GE, Hovell MF, Zakarian JM, Bernert JT, Pirkle JL, Hammond SK. Measuring environmental tobacco smoke exposure in babies: the reliability and validity of motherreports in a sample of low-income families. Health Psychol In press. 